\title{
The GALEN rhinosinusitis cohort: chronic rhinosinusitis with nasal polyps affects health-related quality of life*
}

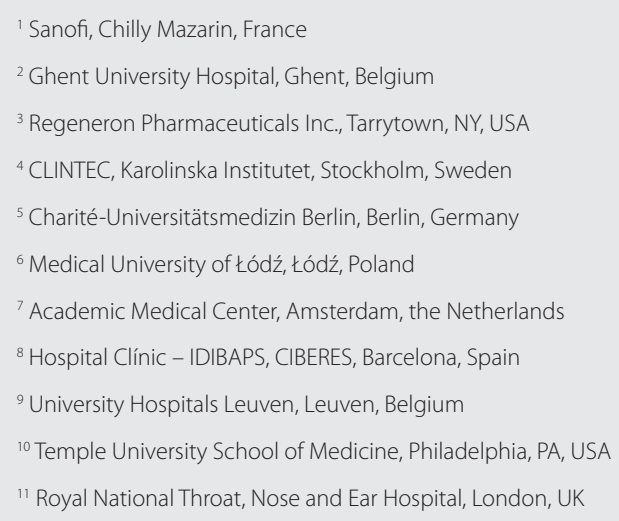

Asif Khan ${ }^{1,2}$, Thi Minh Thao Huynh', Griet Vandeplas², Vijay N. Joish ${ }^{3, * *}$, Leda P. Mannent ${ }^{1}$, Peter Tomassen ${ }^{2}$, Thibaut van Zele ${ }^{2}$, Lars-Olaf Cardell ${ }^{4}$, Julia Arebro ${ }^{4}$, Heidi Olze ${ }^{5}$, Ulrike Förster-Ruhrmann ${ }^{5}$, Marek L. Kowalski ${ }^{6}$, Agnieszka Olszewska-Ziąber ${ }^{6 \dagger}$, Wytske Fokkens ${ }^{7}$, Cornelis van Drunen ${ }^{7}$, Joaquim Mullol' ${ }^{8}$, sam Alobid ${ }^{8}$, Peter W. Hellings, ${ }^{9}$, Valérie Hox ${ }^{9}$, Elina Toskala10, Glenis Scadding ${ }^{11}$, Valerie Lund ${ }^{11}$, Claus Bachert ${ }^{2,4}$

Rhinology 57: 0, $000-000,2019$ https://doi.org/10.4193/Rhin19.158

*Received for publication: April 15, 2019

Accepted: May 3, 2019

"Former employee, currently employed at Lexicon Pharmaceuticals, Inc., Basking Ridge, NJ, USA

\section{Abstract}

Background: Chronic rhinosinusitis (CRS) significantly affects health-related quality of life (HRQoL). Few multinational observational studies have evaluated the impact of CRS with nasal polyps (CRSwNP) on patients' HRQoL. This study aimed to assess HRQoL outcomes (including analyses by disease severity and impact of comorbidities and refractory disease) in CRSwNP patients from a large European database.

Methodology: Data were analysed from the Global Allergy and Asthma European Network (GALEN) Rhinosinusitis Cohort, including sociodemographic data, patient-reported disease severity (visual analogue scale), and scores on the 36-Item ShortForm Health Survey (SF-36) questionnaire. Differences in mean SF-36 scores were evaluated between patients with CRSwNP and population norms and between subgroups of interest (disease severity, comorbidity, and refractory disease, defined by a history of sinonasal surgery).

Results: Patients with CRSwNP ( $N=445)$ had significantly lower mean SF-36 Physical Component Summary (PCS) and Mental Component Summary (MCS) scores vs population norms, demonstrating that CRSwNP negatively affects HRQoL. The presence of comorbidities affected HRQoL, as shown by significant differences in PCS scores in patients with asthma or non-steroidal antiinflammatory drug-exacerbated respiratory disease, compared with patients without asthma. Patients with moderate-to-severe disease had significantly lower PCS scores than patients with mild disease. Severe disease had a significant impact on MCS score. History of surgery had a clinically meaningful negative effect on HRQoL compared with no history of surgery.

Conclusions: CRSwNP patients have significantly lower HRQoL compared with population norms. The impact is greater in patients with greater disease severity, comorbidities, or refractory disease.

Key words: nasal polyps, quality of life, rhinitis, sinusitis 


\section{Introduction}

Chronic rhinosinusitis (CRS), an inflammatory condition of the nose and sinuses, is a common medical condition that affects approximately $11 \%$ of adults in Europe ${ }^{(1)}$ and $12 \%$ of adults in the USA ${ }^{(2)}$. CRS has a significant impact on health-related quality of life (HRQoL) ${ }^{(3,4)}$ and is associated with substantial healthcare ${ }^{(5-7)}$ and productivity costs ${ }^{(8)}$. In adults, CRS with or without nasal polyps (CRSwNP and CRSsNP, respectively) is defined in the European Position Paper on Rhinosinusitis and Nasal Polyps (EPOS) guidelines as the presence (for $\geq 12$ weeks) of 2 or more symptoms, 1 of which is either nasal blockage/ obstruction/congestion or nasal discharge (anterior or posterior nasal drip), with facial pain or pressure and/or reduced or lost sense of smell ${ }^{(9)}$. In the majority of patients in Western Europe, CRSwNP is an idiopathic and eosinophilic form of CRS ${ }^{(10)}$ that is characterised by a type 2 helper T cell (Th2) inflammatory profile ${ }^{(11,12)}$. Compared with CRSsNP, CRSwNP is more often associated with symptoms of rhinorrhoea and decreased sense of smell (13) and with comorbid conditions such as asthma and non-steroidal anti-inflammatory drug (NSAID)-exacerbated respiratory disease (N-ERD) ${ }^{(14-16)}$. The prevalence of CRSwNP is estimated between 2.1\% (France) ${ }^{(17)}$ and $4.4 \%$ (Finland) ${ }^{(18)}$ in Europe and $4.2 \%$ in the USA ${ }^{(19)}, 2.5 \%$ in South Korea ${ }^{(20)}$, and $1.1 \%$ in China ${ }^{(21)}$.

There are few large, multinational, observational studies of the burden of CRSwNP on patients' lives or of the impact of disease severity, comorbidities, and refractory disease on HRQOL. The EPOS 2012 guidelines ${ }^{(9)}$ recommend treatment based on disease severity for all adult patients.

The Global Allergy and Asthma European Network (GALEN) is a network of excellence of the leading European clinical and research facilities in allergology and asthma ${ }^{(22)}$. It represents 20 European countries and includes 2 European societies, 60 collaboration centres, 27 research institutes, and 2 small-to-mediumsized enterprises (defined as legally independent companies with $\leq 500$ employees). The GALEN Rhinosinusitis Cohort, a component of the GALEN network, is a European cross-sectional cohort that was established to enhance and advance research on CRS ${ }^{(12,23,24)}$.

The objective of this study was to assess HRQoL outcomes in patients with CRSwNP who participated in the GALEN Rhinosinusitis Cohort. Patients' HRQoL was assessed using the generic HRQoL questionnaire, the 36-Item Short-Form Health Survey (SF-36), and SF-36 scores were compared between patients with CRSwNP and population norms and between patient subgroups. From this large cohort, we were able to assess the impact of CRSWNP and the effect of disease severity, comorbidities (asthma, N-ERD), and refractory disease on patients' HRQoL.

\section{Materials and methods}

Study design

The GALEN Rhinosinusitis Cohort study was a cross-sectional observational study set up within the GALEN consortium to identify inflammatory endotypes, the natural course of the disease, and the impact of the disease on HRQoL in patients with CRS. The study included patient- and clinician-reported data as well as biomarker data from participants. The results of measurement of inflammatory biomarkers have already been published (12). Patients were included from 9 European centres in Belgium, Finland, Germany, the Netherlands, Poland, Spain, Sweden, and the UK between 25 April 2007 and 7 December 2009. The recruitment of patients for the GALEN Rhinosinusitis Cohort and the collection of patient demographic and clinical data have been previously described ${ }^{(23)}$.

\section{Key inclusion criteria}

This analysis focused on adult patients from the GALEN Rhinosinusitis Cohort who had CRSwNP defined according to the EPOS 2007 guidelines ${ }^{(14)}$. Therefore, symptoms had to include nasal blockage/obstruction/congestion or nasal discharge, facial pain or pressure, and reduced or lost sense of smell, and must have been present for $\geq 12$ weeks with endoscopic signs or radiographic changes and without complete resolution.

\section{Patient-reported and clinical outcomes}

In addition to socio-demographic patient information, patientreported outcomes (PROs) and clinical outcomes from the GALEN Rhinosinusitis Cohort database were included in this study. PRO measures included the HRQoL scale, the SF-36 (version 1 ), and the disease severity visual analogue scale (VAS) ${ }^{(14)}$. The SF-36, a generic HRQoL questionnaire, yields an 8-scale profile of functional health and well-being scores ${ }^{(25,26)}$. Its individual domains are physical functioning, role - physical, bodily pain, general health, vitality, social functioning, role - emotional, and mental health ${ }^{(26)}$. The SF-36 also provides psychometrically based physical and mental health summary measures: the Physical Component Summary (PCS) and the Mental Component Summary (MCS) ${ }^{(26)}$. The VAS, a measure of disease severity, was used to assess impairment due to CRS $(0-10 \mathrm{~cm} ; 0=$ no problem, $10=$ worst imaginable problem) ${ }^{(14)}$. Data on previous sinonasal surgeries and comorbidities were documented. The methods for diagnosis of comorbidities in the GALEN Rhinosinusitis Cohort have been described ${ }^{(23)}$. Briefly, a yes/no response from patients was recorded by the investigator for each comorbidity. Allergic status was based on medical history and confirmed by a skinprick test (using the pan-European allergen panel), a blood assay (radioallergosorbent test), or allergen provocation.

\section{Statistical analyses}

Data from patients with CRSwNP and each subgroup (patients with and without comorbidities, with disease of different severities, and with refractory disease) were analysed for descriptive comparison. Qualitative variables are summarised by counts and 
percentages; quantitative variables are summarised by means, medians, and range and standard deviation (SD), with the number of observations for each variable calculated. The denominator for percentages of patients is the total number of observations (N). Study participants with fewer than 2 completed variables were excluded from the analyses. The analyses used observed data. No data imputation was done. Statistical tests of difference were 2-sided, with a global type I error a of 5\% using a Student's t-test of 1 sample for the difference between the mean of a sample and another mean and of 2 samples for the difference between means. The minimal clinically important difference (MCID) was defined according to the SF-36 guidelines: $\geq 2$ points for $\mathrm{PCS}$ and $\geq 3$ points for MCS scores ${ }^{(26)}$. The MCID for each of the 8 individual domains ( $\geq 8.3$ points) was defined using Cohen's rule of thumb ${ }^{(27)}$.

Differences were evaluated using the Student's t-test of 1 sample and the MCID for mean SF-36 scores between patients with CRSwNP and age-adjusted population norms ${ }^{(28)}$. The UK population norm was used because it includes age as a variable. Previous studies have demonstrated that SF-36 questionnaires are similar across European countries, not only in scores but also in structural content ${ }^{(29,30)}$. It was therefore decided that UK population norms were an adequate comparison.

Differences between CRSwNP patients with and without comorbidities (asthma, N-ERD, or both conditions), with different disease severity (VAS), or with refractory disease (those who had undergone previous sinonasal surgery) and population norms were evaluated using the Student's t-test of 1 sample and the MCID for mean SF-36 PCS and MCS scores. Differences in mean SF-36 PCS and MCS scores between CRSwNP patients with and without comorbidities, between patients with and without history of surgery, and in patients with mild, moderate, or severe disease were also evaluated using the Student's t-test of 2 samples and the MCID.

Spider plots ${ }^{(31,32)}$ were used to graphically depict the difference between the group mean score and the adjusted population norm mean for individual domain scores stratified by absence or presence of comorbidity, VAS disease severity score, and history of sinonasal surgery.

The statistical programmes used for the analyses included $R$ and TANAGRA ${ }^{(33,34) \text {. }}$

\section{Results}

\section{Patient demographics and characteristics}

A total of 445 patients with CRSwNP were included from 9 European centres participating in the GALEN network. The demographics and characteristics of the overall population are shown in Table 1. In the overall CRSwNP population, the mean age was 46.8 years; $58.6 \%$ were male; the mean time since diagnosis was 10.5 years; and the mean symptom duration was 12.8 years. The mean disease severity VAS score was 6.4 (SD 2.8),
Table 1. Characteristics of patients in the overall CRSwNP population.

\begin{tabular}{|lcc|}
\hline Characteristic & $\begin{array}{c}\text { Overall CRSwNP } \\
\text { population }(\mathbf{N}=\mathbf{4 4 5})\end{array}$ \\
\hline & $\mathrm{N}^{\mathrm{a}}$ \\
\hline Age, mean $( \pm \mathrm{SD})$, years & 380 & $46.8( \pm 11.2)$ \\
\hline Male, $\mathrm{n}(\%)$ & 379 & $222(58.6)$ \\
\hline Disease severity by VAS, mean $( \pm \mathrm{SD})$ & 341 & $6.4( \pm 2.8)$ \\
\hline Total endoscopic polyp score, & 332 & $3.1( \pm 1.4)$ \\
mean $( \pm \mathrm{SD})$ & 298 & $14.0( \pm 5.5)$ \\
\hline Lund-Mackay CT score, ${ }^{\mathrm{b}}$ mean $( \pm \mathrm{SD})$ & 339 & $12.8( \pm 10.5)$ \\
\hline Symptom duration, mean $( \pm \mathrm{SD})$, years & 320 & $10.5( \pm 10.1)$ \\
\hline Time since diagnosis, mean $( \pm \mathrm{SD})$, years & 338 & $155(45.9)$ \\
\hline Prior sinonasal surgery, $\mathrm{n}(\%)$ & &
\end{tabular}

CRSwNP, chronic rhinosinusitis with nasal polyps; CT, computed tomography; $n$, number of subjects in subgroup; $N$, number of subjects overall; $\mathrm{SD}$, standard deviation; VAS, visual analogue scale.

${ }^{a}$ Number observed. ${ }^{b}$ Lund-Mackay CT score ranges from 0 to 12 on each side; $0=$ no abnormalities and $12=$ complete opacification of each sinus system and occlusion of the ostiomeatal complex ${ }^{(35)}$.

and almost half of the CRSwNP patients ( $n=155 / 338 ; 45.9 \%$ )

had undergone previous sinonasal surgery. The methodology and descriptive results of the GALEN Rhinosinusitis Cohort study have been described by Khan and colleagues ${ }^{(23)}$.

\section{Impact of CRSwNP on HRQoL}

Compared with population norms, patients with CRSwNP had significantly lower mean SF-36 PCS and MCS scores $(p<0.0001)$ (Figure 1A). The differences exceeded the MCID for both PCS and MCS scores, demonstrating that CRSwNP has a clinically meaningful negative impact on HRQoL. Across all 8 individual SF-36 domains, patients with CRSwNP had significantly lower mean scores than population norms (all $p<0.0001$ ) (Figure 1B). These differences were clinically meaningful (MCID $\geq 8.3$ points) in 5 of the 8 domains: role - physical $(-18.6)$, bodily pain $(-11.6)$, general health $(-16.8)$, social functioning $(-15.5)$, and role emotional (-15.1).

\section{Impact of asthma and N-ERD on HRQoL}

Patients with CRSwNP and comorbidities such as asthma ( $\mathrm{n}=$ $135)$ or N-ERD $(n=11)$ had significantly lower mean SF-36 PCS (with asthma $\mathrm{p}<0.0001$; with N-ERD $\mathrm{p}<0.001$ ) and MCS scores (with asthma $\mathrm{p}<0.0001$; with N-ERD $\mathrm{p}=0.014$ ) compared with population norms, and the differences between groups were clinically meaningful (PCS difference -6.9 for patients with asthma, -9.2 for patients with N-ERD; MCS difference -4.4 for patients with asthma, -7.2 for patients with N-ERD) (Figure 2A). Compared with CRSwNP patients without asthma ( $n=147)$, 
(A)

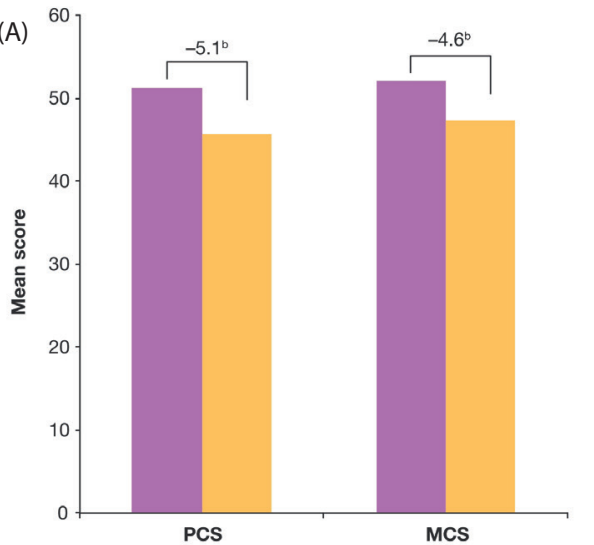

(B)

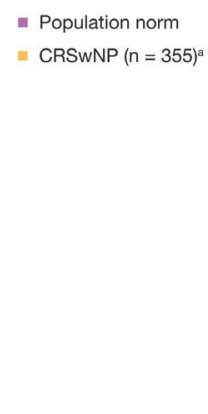

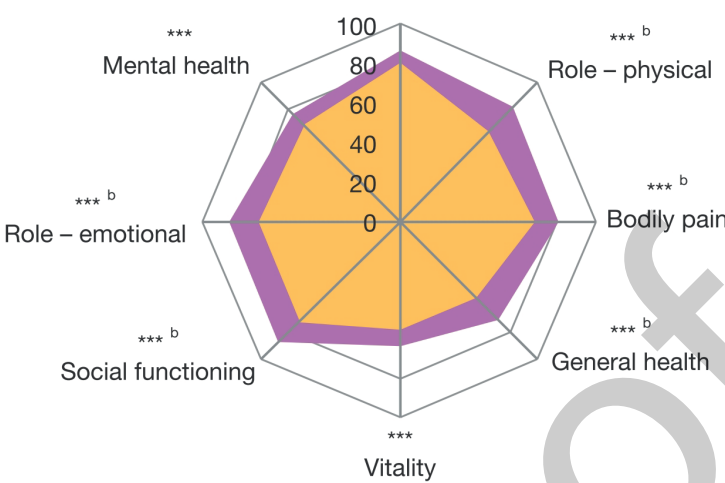

Figure 1. (A) Mean SF-36 Physical Component Summary (PCS) and Mental Component Summary (MCS) scores for patients with CRSwNP vs ageadjusted population norms. Values above bars indicate the difference in scores between age-adjusted population norms and patients with CRSwNP;

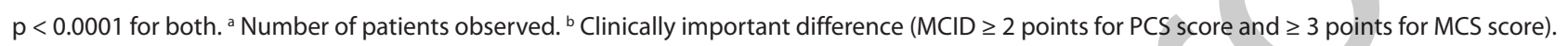
(B) Mean SF-36 domain scores for CRSwNP patients vs age-adjusted population norms. a Number of patients observed. ${ }^{b} S c o r e$ difference between CRSwNP and the population norm $>$ MCID. ${ }^{* * *} p<0.0001$. CRSwNP, chronic rhinosinusitis with nasal polyps; MCID, minimum clinically important difference; SF-36, 36-Item Short-Form Health Survey.

(A)

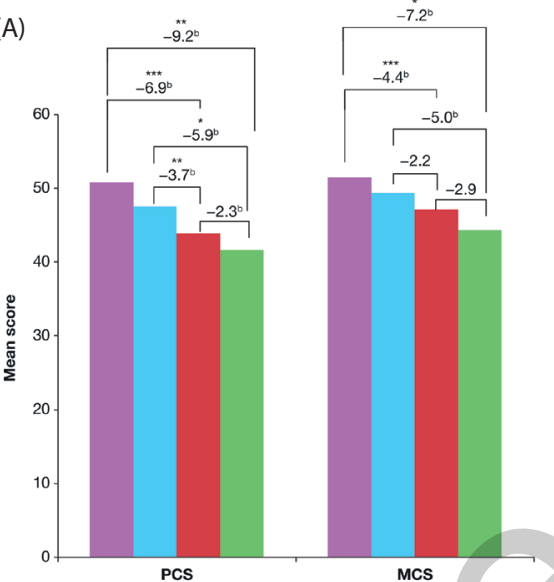

(B)

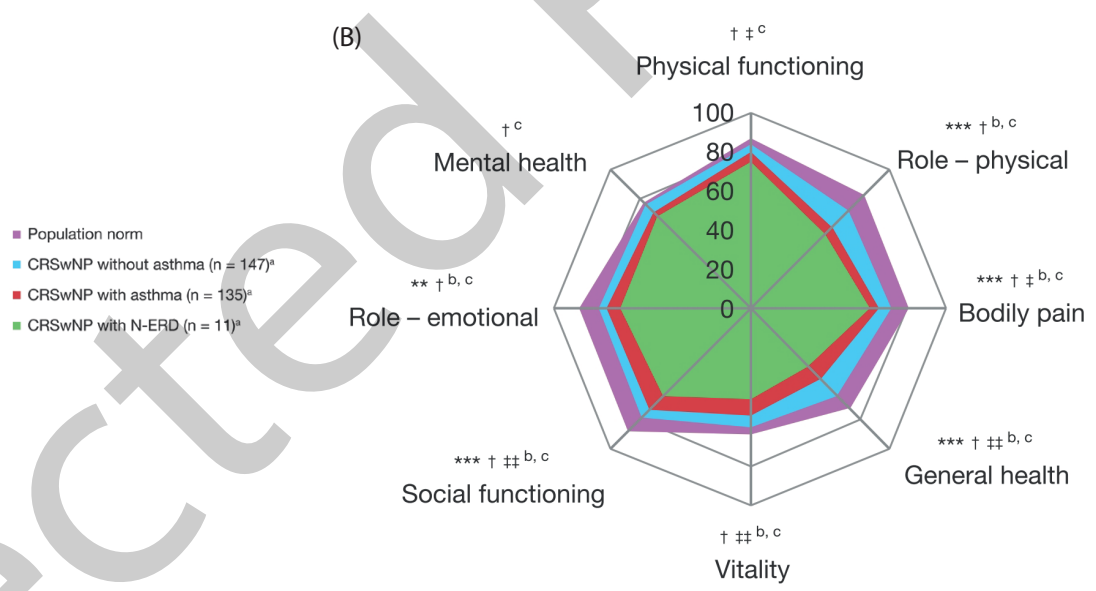

Figure 2. (A) Mean SF-36 Physical Component Summary (PCS) and Mental Component Summary (MCS) scores for patients with CRSwNP without asthma, patients with CRSwNP with asthma or NSAID-exacerbated respiratory disease (N-ERD), and age-adjusted population norms. Values above

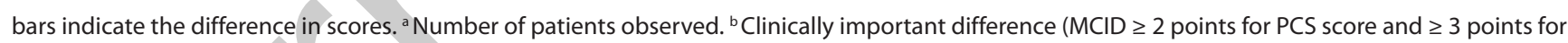
MCS score). ${ }^{*} \mathrm{p}<0.05 ;{ }^{* *} \mathrm{p}<0.01$; ${ }^{* * *} \mathrm{p}<0.0001$. (B) Mean SF-36 domain scores for CRSwNP patients without asthma, CRSwNP patients with asthma or N-ERD, and age-adjusted population norms. ${ }^{a}$ Number of patients observed. ${ }^{\text {b }}$ Score difference between CRSwNP with asthma and population norms > MCID. c Score difference between CRSwNP with N-ERD and population norms > MCID. CRSwNP without asthma vs population norms: ** $\leq$ $0.001 ;{ }^{* * *} p \leq 0.0001$. CRSwNP with asthma vs population norms: ${ }^{\dagger} p \leq 0.0001$. CRSwNP with N-ERD vs population norms: ${ }^{\ddagger} p \leq 0.05 ;{ }^{\ddagger \neq} p \leq 0.01$. CRSwNP, chronic rhinosinusitis with nasal polyps; MCID, minimum clinically important difference; NSAID, non-steroidal anti-inflammatory drug; SF-36, 36-Item Short-Form Health Survey.

CRSwNP patients with comorbid asthma had a significantly lower PCS score $(p=0.002)$; this difference was also clinically meaningful (-3.7). The presence of comorbid N-ERD also decreased the PCS score; this difference was statistically significant $(p=0.031)$ and clinically meaningful $(-5.9)$ compared with patients with CRSwNP without asthma (Figure 2A). Compared with CRSwNP patients with asthma, CRSwNP patients with N-ERD had lower PCS scores; this difference was, however, not statis- tically significant $(p=0.484)$ (Figure $2 A)$.

Compared with patients with CRSwNP without asthma, patients with CRSwNP and comorbid asthma or N-ERD had lower MCS scores, although not statistically significantly so. The MCS score difference for patients with asthma was not clinically meaningful (-2.2). By contrast, the MCS score difference for patients with $\mathrm{N}$-ERD was clinically meaningful (-5.0) (Figure 2A).

Across all 8 individual SF-36 domains, patients with CRSWNP and 

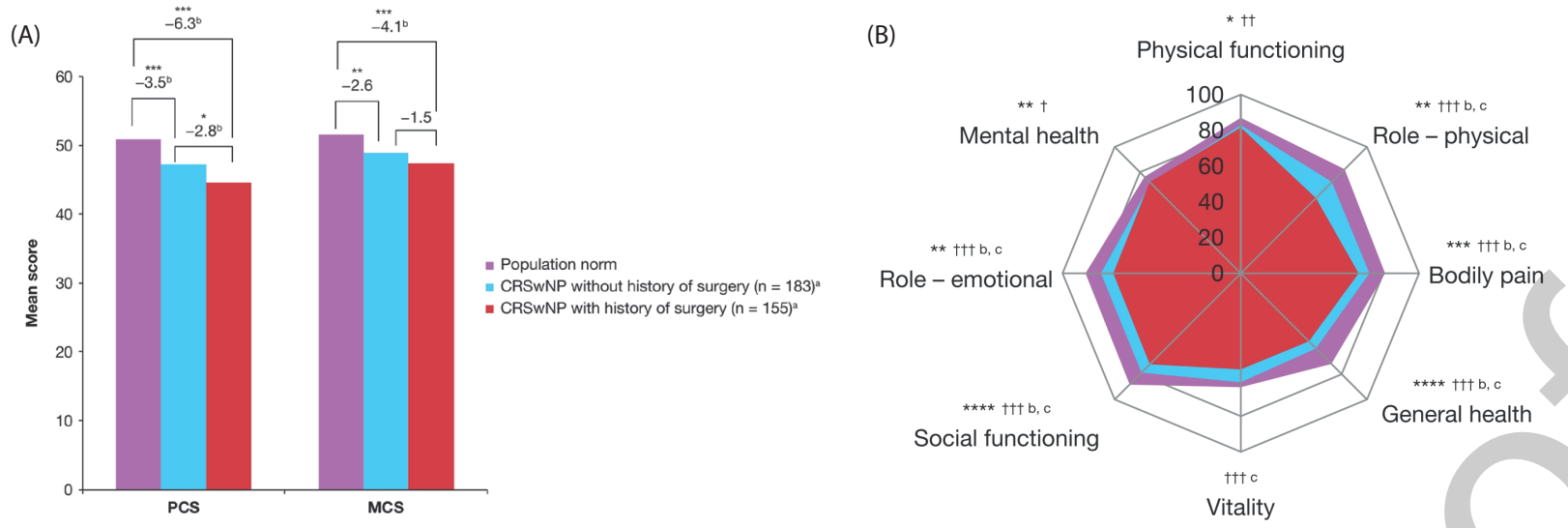

Figure 3. (A) Mean SF-36 Physical Component Summary (PCS) and Mental Component Summary (MCS) scores for patients with CRSwNP without history of surgery, patients with CRSwNP with history of surgery, and age-adjusted population norms. Values above bars indicate the difference in scores. ${ }^{a}$ Number of patients observed. ${ }^{b}$ Clinically important difference (MCID $\geq 2$ points for $\mathrm{PCS}$ score and $\geq 3$ points for $M C S$ score). ${ }^{*} \mathrm{p}<0.05$; ** $\mathrm{p}<$ $0.01{ }^{* * *} \mathrm{p}<0.0001$. (B) Mean SF-36 domain scores for CRSWNP patients without history of surgery, CRSwNP patients with history of surgery, and age-

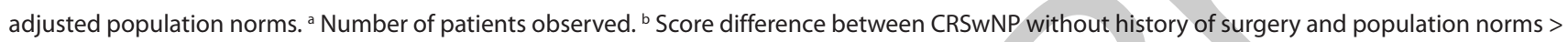
MCID. c Score difference between CRSwNP with history of surgery and populations norms > MCID. CRSwNP without history of surgery vs population norms: ${ }^{*} p \leq 0.05 ;{ }^{* *} p \leq 0.01 ;{ }^{* * *} p \leq 0.001 ;{ }^{* * *} p \leq 0.0001$. CRSwNP with history of surgery vs population norms: ${ }^{\dagger} p \leq 0.01 ;{ }^{+{ }^{+}} p \leq 0.001 ;{ }^{+++} p \leq 0.0001$. CRSWNP, chronic rhinosinusitis with nasal polyps; MCID, minimum clinically important difference; SF-36, 36-Item Short-Form Health Survey.

(A)

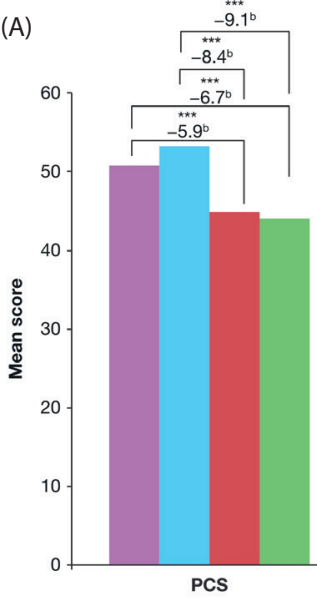

(B)



$* * *+t \neq 0$

Physical functioning
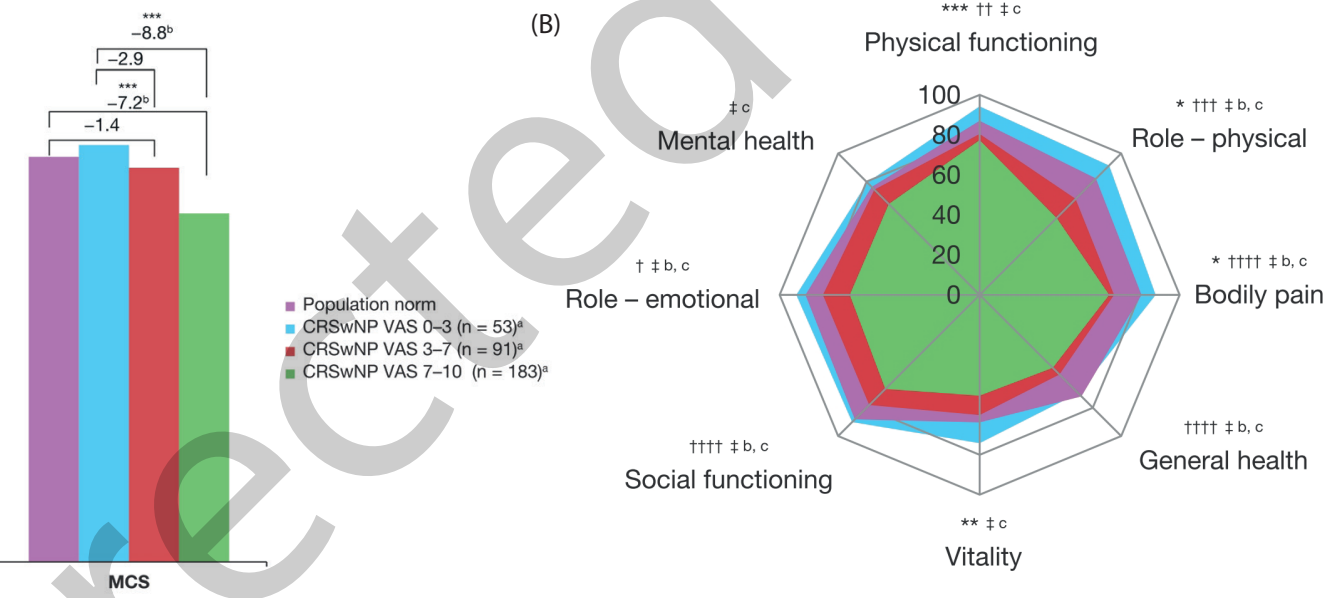

Figure 4. (A) Mean SF-36 Physical Component Summary (PCS) and Mental Component Summary (MCS) scores for patients with mild, moderate or severe CRSWNP and age-adjusted population norms. Values above bars indicate the difference in scores. ${ }^{\mathrm{a}}$ Number of patients observed. ${ }^{\mathrm{b}} \mathrm{Clinically}$ important difference (MCID $\geq 2$ points for PCS score and $\geq 3$ points for MCS score). ${ }^{* *} p<0.0001$. (B) Mean SF-36 domain scores for CRSwNP patients with mild, moderate, or severe disease and age-adjusted population norms. ${ }^{\text {a }}$ Number of patients observed. ${ }^{\mathrm{b}}$ Score difference between CRSWNP VAS 3-7 and population norms > MCID. c Score difference between CRSwNP VAS 7-10 and population norms > MCID. CRSWNP VAS 0-3 vs populations norms: ${ }^{*} p \leq 0.01 ;{ }^{* *} p \leq 0.001 ;{ }^{* * *} p \leq 0.0001$. CRSwNP VAS $3-7$ vs population norms: ${ }^{\dagger} p \leq 0.05 ;{ }^{+t} p \leq 0.001 ;{ }^{+t+} p \leq 0.001 ;{ }^{+++t} p \leq 0.0001$. CRSWNP vs VAS 7-10 norms: ${ }^{\ddagger} p \leq 0.0001$. CRSwNP, chronic rhinosinusitis with nasal polyps; MCID, minimum clinically important difference; SF-36, 36-Item Short-Form Health Survey; VAS, visual analogue scale.

asthma had significantly lower mean scores than population norms (all $p<0.0001$ ). These differences were clinically meaningful ( $M C I D \geq 8.3$ points) in 6 of the 8 domains: role - physical $(-23.3)$, bodily pain $(-15.2)$, general health $(-21.2)$, vitality $(-9.6)$, social functioning (-15.7), and role - emotional (-14.3) (Figure 2B).

Patients with N-ERD had clinically meaningful differences, com- pared with population norms, in all 8 individual SF-36 domains: physical functioning $(-12.0)$, role - physical $(-27.8)$, bodily pain $(-19.4)$, general health $(-29.7)$, vitality $(-17.9)$, social functioning $(-25.6)$, role - emotional (-20.4), and mental health $(-10.0)$. The differences were statistically significant for physical functioning $(p=0.046)$, bodily pain $(p=0.046)$, general health $(p=0.0017)$, vitality $(p=0.0013)$, and social functioning $(p=0.0042)$. 
Impact of refractory CRSwNP on patients' HRQoL, defined by sinonasal surgery history

Sinonasal surgery is normally reserved for patients who are non-responsive to optimal medical treatment or have recur-

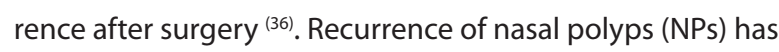
been reported at rates from $15 \%$ to nearly $50 \%$ in the general population during 3 years of follow-up ${ }^{(37)}$.

In this cohort, CRSwNP patients with or without a history of sinonasal surgery had significantly lower mean SF-36 PCS (-6.3 and -3.5 , respectively; $p<0.0001$ for both) and MCS scores $(-4.1$ $[p<0.0001]$ and $-2.6[p=0.005]$, respectively) compared with those of population norms.

Patients with a history of sinonasal surgery $(n=155)$, reflecting refractory disease, had a significantly lower mean SF-36 PCS score $(-2.8 ; p=0.0168)$ than those without a history of surgery $(n=183)$. Differences in MCS scores between patients with and without a history of sinonasal surgery were neither statistically significant nor clinically meaningful (Figure 3A).

Patients with CRSwNP without a history of surgery had significantly lower mean scores than population norms in the following individual SF-36 domains: physical functioning ( $p=$ $0.02)$, role - physical $(p=0.002)$, bodily pain $(p=0.0002)$, role emotional $(p=0.005)$, mental health $(p=0.006)$, general health, and social functioning ( $p<0.0001$ for both) (Figure 3B). These differences were clinically meaningful (MCID $\geq 8.3$ points) in 5 of the 8 domains: role - physical $(-10.0)$, bodily pain $(-8.7)$, general health $(-12.2)$, social functioning $(-9.3)$, and role - emotional (-8.6) (Figure 3B).

Across all 8 individual SF-36 domains, patients with CRSwNP and a history of surgery had significantly lower mean scores than population norms (physical functioning, $p<0.001$; mental health, $p<0.01$; all others, $p<0.0001$ ). These differences were clinically meaningful ( $M C I D \geq 8.3$ points) in 6 of the 8 domains: role - physical (-22.8), bodily pain (-14.8), general health $(-17.9)$, vitality $(-10.0)$, social functioning $(-16.0)$, and role emotional (-15.6) (Figure 3B).

We analysed PCS scores by the number of surgeries as an indicator of disease recurrence. CRSWNP patients with 2 previous surgeries $(n=29)$ saw an incremental effect on PCS score compared with those who had 1 previous surgery $(n=56$; difference -2.6 , $p=0.242)$. In patients with a history of $\geq 3$ surgeries $(n=52)$ compared with those with 1 , there was a further deterioration in PCS score; the impact was both clinically meaningful $(-5.5)$ and statistically significant $(p=0.0042)$.

Impact of VAS disease severity on CRSwNP patients' HRQoL CRSwNP patients with a VAS severity score of 0-3 (mild disease, $\mathrm{n}=53$ ) had mean PCS and MCS scores similar to or higher than those of population norms (Supplementary Table 1). CRSwNP patients with a VAS score of 3-7 (moderate disease, $n=91$ ) had lower mean PCS and MCS scores than population norms; the difference was statistically significant for the PCS score ( $p$ $<0.00001$; Supplementary Table 1). Patients with a VAS score of 7-10 (severe disease, $n=183$ ) had significantly lower mean PCS and MCS scores compared with population norms (all $p<$ 0.00001; Supplementary Table 1).

Compared with patients with mild disease, patients with moderate or severe disease had lower PCS and MCS scores. The differences in PCS scores were statistically significant and clinically meaningful (moderate vs mild -8.4 , severe vs mild $-9.1 ; p<0.0001$ for both). The difference in MCS scores between patients with moderate disease and those with mild disease was not clinically meaningful (-2.9) or statistically significant. By contrast, the difference in MCS scores between patients with severe disease and those with mild disease was clinically meaningful $(-8.8)$ and statistically significant $(p<0.0001)$ (Figure $4 A$ ).

Compared with population norms, CRSwNP patients with mild disease (VAS score of 0-3) had similar or higher mean individual domain scores on the SF-36 (Figure 4B; Supplementary Table 1), and patients with moderate disease (VAS score 3-7) had lower individual SF-36 domain scores. These differences were clinically meaningful for all domains except for physical function, vitality, and mental health. The differences were statistically significant for 6 of the 8 individual domains: physical functioning $(p<0.01)$, role - physical $(p<0.0005)$, bodily pain, general health, social functioning (all $p<0.0001$ ), and role - emotional $(p=0.0128$ ) (Figure 4B; Supplementary Table 1). Patients with severe disease (VAS score 7-10) had lower individual domain scores compared with population norms; the differences were statistically significant and clinically meaningful for all 8 individual domains $(\mathrm{p}<$ 0.00001) (Figure 4B; Supplementary Table 1).

\section{Discussion}

This analysis, performed on a large, multinational, cross-sectional patient cohort, demonstrated that patients with CRSwNP have significantly worse HRQoL than population norms, as shown by the significantly lower scores in the CRSwNP population for all 8 domains of the SF-36.

The presence of comorbidities affects HRQoL, as shown by the significant and clinically meaningful differences in PCS score between patients with asthma or N-ERD and patients without asthma. Although not significantly so, the MCS scores of CRSwNP patients with comorbid asthma or N-ERD were lower than those of patients without asthma. This difference was only clinically relevant in patients with comorbid N-ERD.

A history of sinonasal surgery (an indicator of refractory or recurrent CRSwNP), compared with no history of sinonasal surgery, has a clinically meaningful negative impact on HRQoL. However, despite undergoing surgery, patients still experienced recurrence; a criterion for inclusion in the study was the presence of symptoms for $\geq 12$ weeks without complete resolution. Therefore, the negative impact of a history of surgery on these 
patients' $\mathrm{HRQ}$ L was probably due to the fact that symptom recurrence after surgery is a marker of more severe disease. $\mathrm{HRQ}$ L is also affected by disease severity, as demonstrated by statistically significant and clinically meaningful lower PCS scores of patients with moderate or severe disease compared with patients with mild disease. MCS scores were also significantly lower, with a clinically meaningful difference, but only in patients with severe CRSWNP compared with those with mild CRSwNP.

This study focused on HRQoL in patients with CRSwNP because these patients are often reported to have a higher disease burden than those with CRSsNP ${ }^{(38,39)}$. NP disease, as described here, includes chronic inflammation of the nose and sinuses (CRS). The negative impact of CRSwNP on patients' HRQoL shown in this study is consistent with that reported for CRS in other studies ${ }^{(3,38,40-43)}$. Philpott et al. ${ }^{(43)}$ studied 1470 UK patients (1249 CRS patients and 221 controls) and reported that CRS is associated with significant impairment of quality of life, as demonstrated by the highly significant differences observed in SF-36 and 22-item Sino-Nasal Outcome Test (SNOT-22) scores between CRS patients and controls. Alobid et al. ${ }^{(44)}$ studied 130 Spanish patients with NPs and reported that non-asthmatic patients with NP have significantly better HRQoL than asthmatic patients with $\mathrm{NP}$, with respect to their SF-36 scores for the individual domains physical functioning, role - physical, bodily pain, and vitality. Aspirin sensitivity (N-ERD), compared with aspirin tolerance and NPs, was not reported to have an additional negative impact on HRQoL ${ }^{(44)}$.

The potential limitations of the present study need to be taken into consideration: this was not an epidemiological study, and the fact that participating centres were large tertiary institutions may have led to an inclusion bias towards patients with higher disease burden and more severe CRSwNP. In addition, as in any questionnaire-based study, the results may have been subject to recall bias. The GALEN Rhinosinusitis Cohort study recruited 187 adults with no history of CRS symptoms ${ }^{(23)}$, but these patients were not considered an appropriate control group as they were outpatients of ear, nose, and throat clinics of centres participating in the GALEN and, as such, would have had other nasal or sinus disorders. Although the use of age-adjusted UK population norms as controls instead of healthy patients is a potential limitation of our study, previous studies have demonstrated that the SF-36 results are similar across European countries, not only with respect to scores but also to structure ${ }^{(29,30)}$. Self-assessed health status measured by the SF-36 resulted in similar scores in 3 European national surveys (UK, Germany, and Italy), indicating the comparability of content of the SF-36 in different translations ${ }^{(29)}$.

A comparison of algorithms used to rate PCS and MCS scores (PCS-36/MCS-36) based on the SF-36 from the International Quality of Life Assessment project (across 10 different coun- tries) indicated a high degree of similarity within each country between standard and country-specific algorithms ${ }^{(30)}$. In the absence of age-based SF-36 data from each of the participating European countries, we deemed the UK population norms to provide an adequate comparison. However, of the 8 European countries encompassing the GALEN (Belgium, Finland, Germany, Netherlands, Poland, Spain, Sweden, and the UK), only 5 were included in the comparative analysis by Ware et al. ${ }^{\left({ }^{30}\right)}$. Three countries, therefore, did not have data that have been shown to be normative.

The strength of the present study is that it consisted of a large cross-sectional cohort of patients recruited throughout Europe and for whom many clinical data and biomarkers ${ }^{(12)}$ are available. Given the lack of large, multinational, observational studies of the burden of CRSwNP in patients' lives, this study may serve researchers in assessing the impact of CRSWNP on HRQoL in detail.

\section{Conclusion}

This analysis, performed on a large cross-sectional patient cohort, confirmed that patients with CRSwNP have significantly lower $\mathrm{HRQ}$ oL than population norms. Compared with population norms and patients without comorbidities, CRSwNP patients with comorbidities (asthma, N-ERD) had worse SF-36 PCS and MCS scores; these between-group differences were statistically significant and clinically meaningful. A higher VAS category (greater disease severity) was associated with a greater negative impact on HRQoL. The number of previous surgeries had a clinically meaningful negative impact on HRQoL. PROs may be more useful than objective measures for assessing the impact of CRSwNP on patients' HRQoL.

\section{Acknowledgements}

Research sponsored by Sanofi and Regeneron Pharmaceuticals, Inc. Medical writing/editorial assistance provided by Ferdinando Giacco, PhD, and Ronald van Olffen, PhD, of Excerpta Medica, funded by Sanofi Genzyme and Regeneron Pharmaceuticals, Inc.

\section{Authorship contribution}

AK contributed to the protocol development and design, assisted with interpretation of the results, reviewed and provided direction for manuscript development, and provided critical feedback and final approval for submission. TMTH and VNJ assisted with analysis, review, and interpretation of the results, reviewed and provided direction for manuscript development, and provided critical feedback and final approval for submission. GV and CB contributed to the protocol development and design, assisted with acquisition and interpretation of the results, reviewed and provided direction for manuscript development, and provided critical feedback and final approval for submission. LPM assisted with interpretation of the results, reviewed and 
provided direction for manuscript development, and provided critical feedback and final approval for submission. PT, TVZ, L-OC, JA, HO, UF-R, MLK, AO-Z, WF, CvD, JM, IA, PWH, VH, ET, GS, and $\mathrm{VL}$ assisted with acquisition and interpretation of the results, reviewed and provided direction for manuscript development, and provided critical feedback and final approval for submission.

\section{Conflict of interest}

AK, TMTH, LPM: employees of Sanofi and may hold stock and/ or stock options in the company. GV, PT, TvZ, L-OC, JA, HO, MLK, AO-Z, PWH, VH: no conflicts of interest to disclose. VNJ: former employee and shareholder of Regeneron Pharmaceuticals, Inc. UF-R: received fees for lectures from Novartis. WF: received research grants from Biolnspire, GSK, Meda Pharma, and Sanofi. CVD: received research grants from Allergopharma, EU, FWO, GSK, and NWO. JM: is or has been a member of national and international scientific advisory boards (consulting) and received fees for lectures and grants for research projects from Allakos, ALK-Abelló, FAES, Genentech (Roche), GSK, Mylan, Meda Pharma, Menarini, MSD, Novartis, Regeneron Pharmaceuticals, Inc., Sanofi Genzyme, UCB, and Uriach Group. IA: consultant for Roche. ET: advisory board member for Allergan. GS: received research grants from ALK and GSK; honoraria for articles, consulting, lectures/chairing, and/or advisory boards from ALK, AstraZeneca, Britannia Pharmaceuticals, Capnia, Church \& Dwight, Circassia, Danone, GSK, Meda, Merck, MSD, Ono Pharmaceutical, Oxford BioTherapeutics, Sanofi-Aventis, Shionogi, UCB, and Uriach Group; travel funding from Bayer and GSK. VL: received paid lectureships from Abbott and MSD, is an advisory board member for Crucell and Johnson \& Johnson, and receives research funding from GSK and Meda Pharma. CB: is or has been a principal investigator for AstraZeneca, GSK, Novartis, and Sanofi.

\section{References}

1. Hastan D, Fokkens WJ, Bachert C, et al. Chronic rhinosinusitis in Europe - an underestimated disease. A GA²LEN study. Allergy 2011; 66: 1216-1223.

2. Hirsch AG, Stewart WF, Sundaresan AS, et al. Nasal and sinus symptoms and chronic rhinosinusitis in a population-based sample. Allergy 2017; 72: 274-281

3. Marambaia PP, Lima MG, Santos KP Gomes Ade M, de Sousa MM, Marques ME. Evaluation of the quality of life of patients with chronic rhinosinusitis by means of the SNOT-22 questionnaire. Braz J Otorhinolaryngol 2013; 79: 54-58.

4. Rudmik L, Smith TL. Quality of life in patients with chronic rhinosinusitis. Curr Allergy Asthma Rep 2011; 11: 247-252.

5. Bhattacharyya N, Orlandi RR, Grebner J, Martinson M. Cost burden of chronic rhinosinusitis: a claims-based study. Otolaryngol Head Neck Surg 2011; 144 440-445

6. Smith KA, Orlandi RR, Rudmik L. Cost of adult chronic rhinosinusitis: a systematic review. Laryngoscope 2015; 125: 1547-1556.

. Van Agthoven M, Uyl-de Groot CA, Fokkens WJ, van de Merwe JP, Busschbach JJ. Cost analysis of regular and filgrastim treatment in patients with refractory chronic rhinosinusitis. Rhinology 2002; 40: 69-74

8. Rudmik L, Smith TL, Schlosser RJ, Hwang $\mathrm{PH}$, Mace JC, Soler ZM. Productivity costs in patients with refractory chronic rhinosinusitis. Laryngoscope 2014; 124: 2007-2012.

9. Fokkens WJ, Lund VJ, Mullol J, et al. European position paper on rhinosinusitis and nasal polyps 2012. Rhinol Suppl 2012; 23: 1-298.

10. Bachert C, Pawankar R, Zhang L, et al. ICON: chronic rhinosinusitis. World Allergy Organ J 2014; $7: 25$

11. Bachert C, Wagenmann M, Hauser U,
Rudack C. IL-5 is upregulated in human nasal polyp tissue. J Allergy Clin Immunol 1997; 99: 837-842

12. Tomassen P, Vandeplas G, van Zele $T$, et al. Inflammatory endotypes of chronic rhinosinusitis based on cluster analysis of biomarkers. J Allergy Clin Immunol 2016; 135: 14491456.

13. Dietz de Loos DA, Hopkins C, Fokkens WJ Symptoms in chronic rhinosinusitis with and without nasal polyps. Laryngoscope 2013; 123: 57-63.

14. Fokkens W, Lund V, Mullol J, et al.; European Position Paper on Rhinosinusitis and Nasal Polyps Group. European position paper on rhinosinusitis and nasal polyps 2007. Rhinol Suppl 2007; 20: 1-136

15. Mullol J, Picado C. Rhinosinusitis and nasal polyps in aspirin-exacerbated respiratory disease. Immunol Allergy Clin North Am 2013; 33: 163-176. Review. Erratum in: Immunol Allergy Clin North Am 2013; 33(3):xi.

16. Kowalski ML, Agache I, Bavbek S, et al. Diagnosis and management of NSAIDexacerbated respiratory disease (N-ERD) - a EAACI position paper. Allergy. 2019; 74: 28-39.

17. Klossek JM, Neukirch F, Pribil C, et al. Prevalence of nasal polyposis in France: a cross-sectional, case-control study. Allergy 2005; 60: 233-237.

18. Hedman J, Kaprio J, Poussa T, Nieminen MM. Prevalence of asthma, aspirin intolerance, nasal polyposis and chronic obstructive pulmonary disease in a populationbased study. Int J Epidemiol 1999; 28: 717 722.

19. Settipane GA, Chafee FH. Nasal polyps in asthma and rhinitis. A review of 6,037 patients. J Allergy Clin Immunol 1977; 59: 17-21.

20. We J, Lee $\mathrm{WH}$, Tan $\mathrm{KL}$, et al. Prevalence of nasal polyps and its risk factors: Korean National Health and Nutrition Examination Survey 2009-2011. Am J Rhinol Allergy 2015; 29: e24-28.

21. Shi JB, Fu QL, Zhang $H$, et al. Epidemiology of chronic rhinosinusitis: results from a cross-sectional survey in seven Chinese cities. Allergy 2015; 70: 533-539.

22. Frew AJ. GA2LEN - The Global Allergy and Asthma European Network. Clin Exp Allergy 2005; 35: 122-125

23. Khan A, Vandeplas G, Huynh TMT, et al. The Global Allergy and Asthma European Network (GALEN) rhinosinusitis cohort: a large European cross-sectional study of chronic rhinosinusitis patients with and without nasal polyps. Rhinology 2019; 57: 32-42.

24. Vandeplas G, Khan A, Huynh TMT, et al. The 'GA2LEN Sinusitis Cohort': an introduction. Clin Transl Allergy 2015; 5 Suppl 4: 01.

25. Lehrer-Coriat E, Mariño-Sánchez F, Alobid I, Mullol J. Quality of life measures in patients on rhinosinusitis trials. Clin Invest 2013; 3: 251-263.

26. Ware JE Jr, Sherbourne CD. The MOS 36-item short-form health survey (SF-36). I. Conceptual framework and item selection. Med Care 1992; 30: 473-483.

27. Cohen J. Statistical Power Analysis for the behavioral sciences. 2nd ed. Hillsdale, NJ: Lawrence Erlbaum Associates, 1988.

28. Bowling $A$, Bond $M$, Jenkinson C, Lamping DL. Short Form 36 (SF-36) Health Survey questionnaire: which normative data should be used? Comparisons between the norms provided by the Omnibus Survey in Britain, the Health Survey for England and the Oxford Healthy Life Survey. J Public Health Med 1999; 21: 255-270.

29. Gandek B, Ware J Jr. Methods for validating and norming translations of health status questionnaires: the IQOLA Project 
approach. International Quality of Life Assessment. J Clin Epidemiol 1998; 51: 953959.

30. Ware JE Jr, Gandek B, Kosinski M, et al. The equivalence of SF-36 summary health scores estimated using standard and country-specific algorithms in 10 countries: results from the IQOLA Project. International Quality of Life Assessment. Clin Epidemiol 1998; 51: 1167-1170.

31. Chambers J, Cleveland W, Kleiner B, Tukey P. Graphical methods for data analysis. Pacific Grove, CA: Wadsworth \& Brooks/Cole, 1983.

32. Strand V, Crawford B, Singh J, Choy E, Smolen JS, Khanna D. Use of "spydergrams" to present and interpret SF-36 health-related quality of life data across rheumatic diseases. Ann Rheum Dis 2009; 68: 1800-1804.

33. R Development Core Team. R: A language and environment for statistical computing. Vienna, Austria: the R Foundation for Statistical Computing; 2011. Available at: www.R-project.org. Accessed November 2018

34. Rakotomalala R. TANAGRA: a free software for research and academic purposes Proceedings of EGC'2005, RNTI-E-3. 2005; 2: 697-702.

35. Lund VJ, Mackay IS. Staging in rhinosinusi- tus. Rhinology 1993; 31: 183-184.

36. Lieberman P, Pattanaik D. Nonallergic rhinitis. Curr Allergy Asthma Rep 2014; 14: 439.

37. Vlaminck $S$, Vauterin $T$, Hellings $P W$, et al. The importance of local eosinophilia in the surgical outcome of chronic rhinosinusitis: a 3 -year prospective observational study. Am J Rhinol Allergy 2014; 28: 260-264.

38. Vaid L, Khanna S, Singh PP. Impact of nasal polyps on quality of life of chronic sinusitis patients. Indian J Otolaryngol Head Neck Surg 2007; 59: 136-141.

39. Banerji A, Piccirillo JF, Thawley SE, et al. Chronic rhinosinusitis patients with polyps or polypoid mucosa have a greater burden of illness. Am J Rhinol 2007; 21: 19-26.

40. Alobid I, Benítez P, Bernal-Sprekelsen M, et al. Nasal polyposis and its impact on quality of life: comparison between the effects of medical and surgical treatments. Allergy 2005; 60: 452-458.

41. Alobid I, Benitez P, Pujols L, et al. Severe nasal polyposis and its impact on quality of life. The effect of a short course of oral steroids followed by long-term intranasal steroid treatment. Rhinology 2006; 44: 8-13.

42. Lange B, Holst R, Thilsing T, Baelum J, Kjeldsen A. Quality of life and associated factors in persons with chronic rhinosinusi- tis in the general population: a prospective questionnaire and clinical cross-sectional study. Clin Otolaryngol 2013; 38: 474-480.

43. Philpott C, Erskine S, Hopkins C, et al. A case-control study of medical, psychological and socio-economic factors influencing the severity of chronic rhinosinusitis. Rhinology 2016; 54: 134-140.

44. Alobid I, Benítez P, Bernal-Sprekelsen M, Guilemany JM, Picado C, Mullol J. The impact of asthma and aspirin sensitivity on quality of life of patients with nasal polyposis. Qual Life Res 2005; 14: 789-793.

Prof. Dr Claus Bachert

Upper Airways Research Laboratory

Department of Otorhinolaryngology

Ghent University Hospital

De Pintelaan 185

9000 Ghent

Belgium

Tel: +32 (0) 93322332

E-mail: claus.bachert@ugent.be

\section{SUPPLEMENTARY DATA}

Supplementary Table 1. SF-36 scores for VAS thresholds in patients with CRSwNP.

\begin{tabular}{|c|c|c|c|c|c|c|c|c|c|c|}
\hline \multirow[t]{2}{*}{ SF-36 domain } & \multirow{2}{*}{$\begin{array}{l}\text { Popula- } \\
\text { tion norm } \\
\text { values } \\
\text { (adjusted } \\
\text { for age) }\end{array}$} & \multirow{2}{*}{$\begin{array}{l}\text { VAS } \\
0-3\end{array}$} & \multirow{2}{*}{$\begin{array}{l}\text { VAS } \\
3-7\end{array}$} & \multirow{2}{*}{$\begin{array}{l}\text { VAS } \\
7-10\end{array}$} & \multicolumn{6}{|c|}{ Difference in mean SF-36 score vs population norm } \\
\hline & & & & & $\begin{array}{c}\text { Difference } \\
\text { VAS 0-3 vs } \\
\text { population } \\
\text { norm }\end{array}$ & p value & $\begin{array}{c}\text { Difference } \\
\text { VAS 3-7 vs } \\
\text { population } \\
\text { norm }\end{array}$ & $p$ value & $\begin{array}{c}\text { Difference } \\
\text { VAS } 7-10 \text { vs } \\
\text { population } \\
\text { norm }\end{array}$ & $p$ value \\
\hline Physical functionin & 87 & 94.2 & 80.4 & 77.4 & 7.25 & $<0.00001$ & -6.57 & 0.0014 & -9.62 & $<0.00001$ \\
\hline Role - physical & 82.3 & 91.5 & 67.9 & 54.4 & 9.21 & 0.0035 & -14.44 & 0.0005 & -27.85 & $<0.00001$ \\
\hline Bodily pain & 80.9 & 88.0 & 67.2 & 64.8 & 7.10 & 0.0011 & -13.70 & $<0.00001$ & -16.12 & $<0.00001$ \\
\hline General health & 71.8 & 69.5 & 56.4 & 51.6 & -2.27 & 0.3924 & -15.39 & $<0.00001$ & -20.16 & $<0.00001$ \\
\hline Vitality & 63.8 & 74.2 & 60.1 & 50.5 & 10.40 & 0.0002 & -3.65 & 0.1245 & -13.31 & $<0.00001$ \\
\hline Social functioning & 88.1 & 90.1 & 78.0 & 66.7 & 1.99 & 0.3305 & -10.08 & 0.0001 & -21.41 & $<0.00001$ \\
\hline Role-emotional & 87.1 & 91.7 & 78.4 & 65.0 & 4.57 & 0.1538 & -8.71 & 0.0128 & -22.12 & $<0.00001$ \\
\hline Mental health & 76.3 & 79.5 & 74.4 & 64.4 & 3.16 & 0.1398 & -1.93 & 0.2790 & -11.92 & $<0.00001$ \\
\hline $\begin{array}{l}\text { Physical Component } \\
\text { Summary }\end{array}$ & 50.8 & 53.3 & 44.9 & 44.1 & 2.46 & 0.0005 & -5.90 & $<0.00001$ & -6.68 & $<0.00001$ \\
\hline $\begin{array}{l}\text { Mental Component } \\
\text { Summary }\end{array}$ & 51.5 & 53.0 & 50.1 & 44.3 & 1.52 & 0.1905 & -1.42 & 0.1823 & -7.24 & $<0.00001$ \\
\hline
\end{tabular}

CRSwNP, chronic rhinosinusitis with nasal polyps; SF-36, 36-Item Short-Form Health Survey; VAS, visual analogue scale. 